\title{
CS-MODULES AND ANNIHILATOR CONDITIONS
}

\author{
MAHMOUD A. KAMAL and AMANY M. MENSHAWY
}

Received 4 June 2002

\begin{abstract}
We study $S$ - $R$-bimodules ${ }_{S} M_{R}$ with the annihilator condition $S=l_{S}(A)+l_{S}(B)$ for any closed submodule $A$, and a complement $B$ of $A$, in $M_{R}$. Such annihilator condition has a direct connection with the CS-condition for $M_{R}$. We make use of this to give a new characterization of CS-modules. Bimodules ${ }_{S} M_{R}$ for which $r_{M} l_{S}(A)=A$ (for every closed submodule $A$ of $M_{R}$ ) are also dealt with. Such modules are called $W^{*}$-modules. We give the extra added annihilator conditions to $W^{*}$-modules to be equivalent to the continuous (quasicontinuous) modules.
\end{abstract}

2000 Mathematics Subject Classification: 16D80.

1. Introduction. Let $R$ and $S$ be rings and let ${ }_{S} M_{R}$ be a bimodule. For any $X \leq M$ and $T \leq S$, write $l_{S}(X)=\{s \in S: s X=0\}$ and $r_{M}(T)=\{m \in M: T m=$ $0\}$. Let $\lambda: S \rightarrow \operatorname{End}\left(M_{R}\right)$ be the canonical ring homomorphism. For each $s \in S$, we identify $\lambda(s)$ with $s$. A submodule $A$ is essential in $M$ (denoted by $A \leq^{e} M$ ) if $A \cap B \neq 0$ for every nonzero submodule $B$ of $M$. A submodule $A$ is closed in $M$ if it has no proper essential extensions in $M . A \leq^{\oplus} M$ signifies that $A$ is a direct summand of $M$ (or simply a summand). A module $M$ is called a CS-module if every closed submodule of $M$ is a summand. The module $M$ is continuous if it is a CS-module and satisfies condition $\left(\mathrm{C}_{2}\right)$ : if $A \cong B \leq M$ with $A \leq{ }^{\oplus} M$, then $B \leq{ }^{\oplus} M$. A generalization of condition $\left(\mathrm{C}_{2}\right)$ is $\left(\mathrm{GC}_{2}\right)$ (see [4]): if $A$ is a submodule of $M$ with $A \cong M$, then $A \leq{ }^{\oplus} M$. The module $M$ is quasicontinuous if it is a CS-module and satisfies condition $\left(\mathrm{C}_{3}\right)$ : if $A, B \leq{ }^{\oplus} M$ with $A \cap B=0$, then $A \oplus B \leq \leq^{\oplus} M$. It is known that $M$ is quasicontinuous if and only if $M=$ $A \oplus B$ whenever $A$ and $B$ are complements of each other in $M$ (see [3, Theorem 2.8]).

Camillo et al. [1] have dealt with Ikeda-Nakayama rings that are related to continuous and quasicontinuous rings.

For a bimodule ${ }_{S} M_{R}$, Wisbauer et al. [4] have studied the annihilator condition $l_{S}(A \cap B)=l_{S}(A)+l_{S}(B)$ for any submodules $A$ and $B$ of $M_{R}$, and the condition $S=l_{S}(A)+l_{S}(B)$ for any submodules $A$ and $B$ of $M_{R}$ with $A \cap B=0$. Consequently, they obtained new characterizations of quasicontinuous modules. We adapt their ideas here to study a variation of the above annihilator condition which is connected to CS-modules, and obtain a new characterization of CS-modules in Section 2. 
In Section 3, we study the bimodules ${ }_{S} M_{R}$ which satisfy the following condition:

$$
S=l_{S}(A)+l_{S}(B)
$$

for any two relative complements $A$ and $B$ in $M_{R}$. Such modules are clearly quasicontinuous modules, while there are quasicontinuous modules which do not satisfy condition (1.1). For example, consider $R$ as a commutative integral domain with field of quotients $Q$ and let $M=Q \oplus Q$. In Lemma 3.2, we give a necessary and sufficient condition for quasicontinuous modules to satisfy condition (1.1). In the case of $S=\operatorname{End}\left(M_{R}\right)$, every quasicontinuous module must have condition (1.1). As a generalization of this condition, we introduce the concept of $W^{*}$-modules (bimodules ${ }_{S} M_{R}$ for which $A=r_{M} l_{S}(A)$ for every closed submodule $A$ of $M_{R}$ ). It is clear that any bimodule with condition (1.1) is a $W^{*}$-module, while in general the converse is not true. Proposition 3.8 indicates when a $W^{*}$-module satisfies condition (1.1).

In Section 4, we discuss the equivalence between $W^{*}$-modules and continuous (quasicontinuous) modules over an arbitrary ring $S$. Then we draw the consequences when $S$ is the endomorphism ring of $M_{R}$.

2. CS-modules and annihilator conditions. The proofs of the lemmas and propositions, presented in this section, are adaptations of the arguments in [4].

LEMMA 2.1. Let ${ }_{S} M_{R}$ be a bimodule. If for every closed submodule $A$ of $M_{R}$ there exists a complement $B$ of $A$ in $M_{R}$ such that $S=l_{S}(A)+l_{S}(B)$, then $M_{R}$ is a CS-module.

Proof. Let $A$ be a closed submodule of $M_{R}$. Then by assumption there exists a complement $B$ of $A$ in $M_{R}$ such that $S=l_{S}(A)+l_{S}(B)$. Write $1_{S}=$ $u+v$, where $u \in l_{S}(A)$ and $v \in l_{S}(B)$. It follows that $a=v a$ for all $a \in A$, $b=u b$ for all $b \in B$, and $v B=u A=0$. Thus $B \subseteq r_{M}(v) \subseteq r_{M}\left(v^{2}\right)$ and $r_{M}\left(v^{2}\right) \cap$ $A=0$. Since $B$ is a complement of $A$ in $M_{R}$, we have $B=r_{M}(v)=r_{M}\left(v^{2}\right)$. Similarly, $A=r_{M}(u)=r_{M}\left(u^{2}\right)$. Now we show that $(v u) M=0$. Let $v u m=$ $a+b$, where $m \in M, a \in A$, and $b \in B$. Noting that $v u=u v$, we have that $\left(v^{2} u^{2}\right) m=(v u)(a+b)=0$. Hence $u^{2} m \in r_{M}\left(v^{2}\right)=r_{M}(v)$, and this gives that $u^{2} v m=v u^{2} m=0$. Then $v m \in r_{M}\left(u^{2}\right)=r_{M}(u)$; and thus $v u m=u v m=0$. So $(v u) M \cap(A+B)=0$. Since $A+B$ is essential in $M_{R}$, $(v u) M=0$. So $u M \subseteq$ $r_{M}(v)=B$ and $v M \subseteq r_{M}(u)=A$ and hence $M=v M+u M=A+B=A \oplus B$. Therefore $A$ is a summand of $M_{R}$.

REMARK 2.2. The converse of Lemma 2.1 is not true. For example, there are torsion-free CS-modules over commutative integral domains, which do not satisfy the given condition in Lemma 2.1.

The next lemma follows from [4, Lemma 3]. 
LEMMA 2.3. Let ${ }_{S} M_{R}$ be a bimodule, where ${ }_{S} M$ is faithful, and let $M_{R}=A \oplus B$. If the projection $f$ of $M$ onto $A$ along $B$ is given by $f(m)=s m$ for some $s \in S$, and all $m \in M$, then $S=l_{S}(A)+l_{S}(B)$.

For any submodules $A$ and $B$ of $M_{R}$ and any $t \in S$, define $\alpha_{t}: A+B \rightarrow M$, $a+b \rightarrow t a$ (see [4]).

Proposition 2.4. Let ${ }_{S} M_{R}$ be a bimodule such that ${ }_{S} M$ is faithful. The following are equivalent:

(1) $M_{R}$ is $C S$ and for any $f^{2}=f \in \operatorname{End}\left(M_{R}\right)$, there exists $s \in S$ such that $f(m)=s m$, for all $m \in M_{R}$;

(2) for every closed submodule $A$ of $M_{R}$, there exists a complement $B$ of $A$ in $M_{R}$ such that $S=l_{S}(A)+l_{S}(B)$;

(3) for every closed submodule $A$ of $M_{R}$, there exists a complement $B$ of $A$ in $M_{R}$ such that $S=l_{S}(A) \oplus l_{S}(B)$;

(4) for every closed submodule $A$ of $M_{R}$, there exists a complement $B$ of $A$ in $M_{R}$ such that for every $t \in S$, the diagram

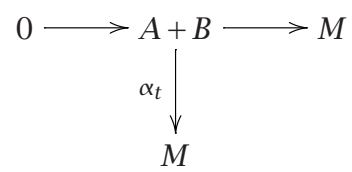

can be extended by $\lambda(s)$, for some $s \in S$.

Proof. $(1) \Rightarrow(2)$. Let $A$ be a closed submodule of $M_{R}$. Since $M_{R}$ is a CSmodule, there exists $f^{2}=f \in \operatorname{End}\left(M_{R}\right)$ such that $A=f M$. By (1), there exists $s \in S$ such that $f(m)=s m$, for all $m \in M_{R}$. Hence $\left(s^{2}-s\right) M=\left(f^{2}-f\right) M=0$. Since ${ }_{S} M$ is faithful, it follows that $s$ is an idempotent in $S$. Now we have

$$
l_{S}(A)=l_{S}(f M)=l_{S}(s M)=l_{S}(s)=S(1-S) .
$$

Similarly, $l_{S}(B)=S_{S}$, where $B=:(1-f) M$. Thus $S=l_{S}(A)+l_{S}(B)$.

$(2) \Rightarrow(1)$. It is clear by Lemma 2.1 that $M_{R}$ is CS. Now let $f^{2}=f \in \operatorname{End}\left(M_{R}\right)$, and denote $A=f(M)$. By (2), there exists a complement $B$ of $A$ in $M_{R}$ such that $S=l_{S}(A)+l_{S}(B)$. The argument of the proof of Lemma 2.1 shows that $M=A \oplus B$. Let $\pi$ be the projection of $M$ onto $A$ along $B$. Then

$$
l_{S}(A)=l_{S}(\pi M)=\{s \in S: s \pi=0\}
$$

(by considering $s$ the homomorphism given by left multiplication by $s$ ) and

$$
l_{S}(B)=l_{S}((1-\pi) M)=\{s \in S: s(1-\pi)=0\} .
$$


Let $1=s^{\prime}+s$, where $s^{\prime} \in l_{S}(A)$ and $s \in l_{S}(B)$. Thus $s^{\prime} \pi=0$ and $s(1-\pi)=0$. It follows that $0=s(1-\pi)=\left(1-s^{\prime}\right)(1-\pi)=1-\pi-s^{\prime}$. Therefore $f(m)=$ $\pi(m)=s m$ for all $m \in M$.

(2) $\Rightarrow(3)$. From the argument in the proof of Lemma 2.1, we have $M=A \oplus B$. Since ${ }_{S} M$ is faithful, we have $0=l_{S}(M)=l_{S}(A+B)=l_{S}(A) \hbar l_{S}(B)$ and hence $S=l_{S}(A) \oplus l_{S}(B)$.

(3) $\Rightarrow(4)$. Let $A$ be a closed submodule of $M_{R}$. By (3), there exists a complement $B$ of $A$ such that $S=l_{S}(A) \oplus l_{S}(B)$. Write $t=u+v$, where $u \in l_{S}(A)$ and $v \in l_{S}(B)$. Then $\alpha_{t}(a+b)=t a=(u+v) a=v a=v(a+b)=\lambda(v)(a+b)$.

$(4) \Rightarrow(2)$. Let $A$ be a closed submodule of $M_{R}$. By (4), there exists a complement $B$ of $A$ in $M_{R}$ satisfying diagram (2.1). By (4), there exists $s \in S$ such that $\lambda(s)$ extends $\alpha_{t}$. Thus, for all $a \in A$ and $b \in B, t a=\alpha_{t}(a+b)=\lambda(s)(a+b)=$ $s(a+b)$. It follows that $(1-s) a+(-s) b=0$, for all $a \in A$ and $b \in B$. So $1-s \in l_{S}(A)$ and $-s \in l_{S}(B)$ and hence $1=(1-s)-(-s) \in l_{S}(A)+l_{S}(B)$. Therefore $S=l_{S}(A)+l_{S}(B)$.

COROLLARY 2.5. The following are equivalent for a bimodule ${ }_{S} M_{R}$ with $S=$ $\operatorname{End}\left(M_{R}\right)$ :

(1) $M_{R}$ is a CS-module;

(2) for every closed submodule $A$ of $M_{R}$, there exists a complement $B$ of $A$ in $M_{R}$ such that $S=l_{S}(A)+l_{S}(B)$;

(3) for every closed submodule $A$ of $M_{R}$, there exists a complement $B$ of $A$ in $M_{R}$ such that $S=l_{S}(A) \oplus l_{S}(B)$;

(4) for every closed submodule $A$ of $M_{R}$, there exists a complement $B$ of $A$ in $M_{R}$ such that for every $t \in S$, diagram (2.1) can be extended by some $g: M \rightarrow M$.

PROPOSITION 2.6. Let $S$ be the center of $\operatorname{End}\left(M_{R}\right)$. The following are equivalent:

(1) for every closed submodule $A$ of $M_{R}$, there exists a complement $B$ of $A$ in $M_{R}$ such that $S=l_{S}(A)+l_{S}(B)$;

(2) $M_{R}$ is $C S$ and every idempotent of $\operatorname{End}\left(M_{R}\right)$ is central;

(3) $M_{R}$ is $C S$ and every closed submodule of $M_{R}$ is fully invariant.

ProOF. $\quad(1) \Leftrightarrow(2)$ by Proposition 2.4 .

$(2) \Rightarrow(3)$. Let $A$ be a closed submodule of $M$. By CS, $A$ is a direct summand of $M_{R}$. Then $A=f(M)$ for some $f^{2}=f \in \operatorname{End}\left(M_{R}\right)$. For any $g \in \operatorname{End}_{R}(M)$, since $f$ is central by (2), $g(A)=g(f(M))=f(g(M)) \subseteq f(M)=A$. This shows that $A$ is a fully invariant submodule of $M$.

(3) $\Rightarrow(2)$. Let $f, g \in \operatorname{End}_{R}(M)$ with $f^{2}=f$. Therefore $f(M)$ is a closed submodule of $M_{R}$. By $(3), g(f(M)) \subseteq f(M)$ and $g((1-f)(M)) \subseteq(1-f)(M)$. It follows that $f g f=g f$ and $(1-f) g(1-f)=g(1-f)$. Thus, $g-g f=g(1-f)=$

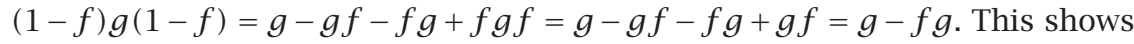
that $f g=g f$. 
3. Condition (1.1) and its generalizations. The next lemma is clear.

LEMMA 3.1. The following are equivalent for a bimodule ${ }_{S} M_{R}$ :

(1) $S=l_{S}(A)+l_{S}(B)$ for any two relative complements $A$ and $B$ of $M_{R}$;

(2) for any submodules $A$ and $B$ of $M_{R}$ with $A \cap B=0, S=l_{S}(A)+l_{S}(B)$.

We say that a bimodule ${ }_{S} M_{R}$ has condition (1.1) if it satisfies one of the equivalent conditions of Lemma 3.1.

The next lemma follows from [4, Lemma 3].

LEMMA 3.2. Let ${ }_{S} M_{R}$ be a bimodule such that ${ }_{S} M$ is faithful. Then the following are equivalent:

(1) M has condition (1.1);

(2) $M$ is quasicontinuous and every idempotent in $\operatorname{End}\left(M_{R}\right)$ is a left multiplication by an element of $S$.

REMARK 3.3 [4, Theorem 8]. In the case of $S=\operatorname{End}\left(M_{R}\right)$, it is clear from Lemma 3.2 that an R-module $M$ is quasicontinuous if and only if $M$ has condition (1.1).

Proposition 3.4. Let ${ }_{S} M_{R}$ be a bimodule which satisfies condition (1.1). Then $A=r_{M} l_{S}(A)$ for all closed submodules $A$ of $M_{R}$.

Proof. Let $A$ be a closed submodule of $M_{R}$ and $B$ a submodule of $r_{M} l_{S}(A)$ such that $A \cap B=0$. By Zorn's lemma, there exists a complement $C$ of $A$ in $M_{R}$ with $B \subseteq C$. By condition (1.1), we have $S=l_{S}(A)+l_{S}(C) \subseteq l_{S}(A)+l_{S}(B)$, so $S=l_{S}(A)+l_{S}(B)$. Since $l_{S}(A)=l_{S} r_{M} l_{S}(A) \leq l_{S}(B)$, it follows that $S=l_{S}(B)$ and hence $B=0$. This shows that $A \leq^{e} r_{M} l_{S}(A)$. Since $A$ is a closed submodule of $M_{R}$, we have $A=r_{M} l_{S}(A)$.

A bimodule ${ }_{S} M_{R}$ is called a $W^{*}$-module if $A=r_{M} l_{S}(A)$ for every closed submodule $A$ of $M_{R}$. It is clear by Proposition 3.4 that every bimodule ${ }_{S} M_{R}$ with condition (1.1) is a $W^{*}$-module. But there are bimodules which are $W^{*}$-modules and do not satisfy condition (1.1). For example, let $S=R=\left[\begin{array}{c}F F \\ 0 F\end{array}\right]$, where $F$ is any field and let $M={ }_{R} R_{R}$. It is clear that $M$ is $W^{*}$-module. But $M_{R}$ is not quasicontinuous, and hence $M$ does not satisfy condition (1.1).

LEMMA 3.5. The following are equivalent for a bimodule ${ }_{S} M_{R}$ :

(1) $A \leq{ }^{e} r_{M} l_{S}(A)$ for all submodules $A$ of $M_{R}$;

(2) ${ }_{S} M_{R}$ is a $W^{*}$-module.

Proof. $\quad(1) \Rightarrow(2)$. This implication is obvious.

(2) $\Rightarrow(1)$. Let $A$ be a submodule of $M_{R}$ and $C$ a maximal essential extension of $A$ in $M_{R}$. We have by (2) that $A \leq{ }^{e} C=r_{M} l_{S}(C)$. Since $r_{M} l_{S}(A) \leq r_{M} l_{S}(C)$, we have $A \leq^{e} r_{M} l_{S}(A)$.

PROPOSITION 3.6. If $S_{S} M_{R}$ is a $W^{*}$-module, then $r_{M}(T)=0$, or $r_{M}(T)$ is uniform for every maximal left ideal $T$ of $S$. 
Proof. Let $T$ be a maximal left ideal of $S$. Since $T \subseteq l_{S} r_{M}(T)$, we have either $l_{S} r_{M}(T)=T$ or $l_{S} r_{M}(T)=S$. If $l_{S} r_{M}(T)=S$, then $r_{M}(T)=0$. If $l_{S} r_{M}(T)=T$, let $N$ be a nonzero submodule of $r_{M}(T)$. Then $T=l_{S} r_{M}(T) \subseteq l_{S}(N) \subseteq S$, and the maximality of $T$ yields $T=l_{S}(N)$. It follows that $r_{M}(T)=r_{M} l_{S}(N)$. Since $M$ is $W^{*}$-module, we have by Lemma 3.5 that $N \leq^{e} r_{M}(T)$. Therefore $r_{M}(T)$ is uniform.

COROLLARY 3.7. Let ${ }_{S} M_{R}$ be a $W^{*}$-module, where every maximal left ideal of $S$ is a left annihilator. Then $r_{M}(T)$ is uniform for every maximal left ideal $T$ of $S$.

Proof. Let $T$ be a maximal left ideal of $S$. From Proposition 3.6, it is enough to show that $r_{M}(T) \neq 0$. Let $r_{M}(T)=0$. By assumption, $T=l_{S} r_{M}(T)=l_{S}(0)=S$, which contradicts the maximality of $T$.

Proposition 3.8. The following are equivalent for a bimodule ${ }_{S} M_{R}$ :

(1) ${ }_{S} M_{R}$ is a $W^{*}$-module and $l_{S}(A)+l_{S}(B)$ is a left annihilator for any two relative complements $A$ and $B$ in $M_{R}$;

(2) ${ }_{S} M_{R}$ has condition (1.1).

Proof. $\quad(1) \Rightarrow(2)$. Let $A$ and $B$ be two relative complements in $M_{R}$. Then by (1), $S=l_{S}(0)=l_{S}(A \cap B)=l_{S}\left(r_{M} l_{S}(A) \cap r_{M} l_{S}(B)\right)=l_{S} r_{M}\left(l_{S}(A)+l_{S}(B)\right)=l_{S}(A)+$ $l_{S}(B)$. Therefore $M$ has condition (1.1).

(2) $\Rightarrow(1)$. This implication is obvious.

4. The relation between $W^{*}$-modules and (quasi-) continuous modules. The following is an immediate consequence of Proposition 3.8.

Proposition 4.1. Let ${ }_{S} M_{R}$ be a bimodule with $S=\operatorname{End}\left(M_{R}\right)$. Then the following are equivalent:

(1) ${ }_{S} M_{R}$ is a $W^{*}$-module and $l_{S}(A)+l_{S}(B)$ is a left annihilator for any two relative complements $A$ and $B$ of $M_{R}$;

(2) $M_{R}$ is quasicontinuous.

Proposition 4.2. Let ${ }_{S} M_{R}$ be a bimodule, where ${ }_{S} M$ is faithful. Then the following are equivalent:

(1) ${ }_{S} M_{R}$ is a $W^{*}$-module, $l_{S}(A)+l_{S}(B)$ is an annihilator for any two relative complements $A$ and $B$ of $M_{R}$, and $M_{R}$ has $G C_{2}$;

(2) $M_{R}$ is a continuous module and every idempotent in $\operatorname{End}\left(M_{R}\right)$ is a left multiplication by an element of $S$.

Proof. $(1) \Rightarrow(2)$. We have by Proposition 3.8 that $M_{R}$ has condition (1.1). Therefore, by Lemma 3.2, $M_{R}$ is a quasicontinuous module. Let $s \in \operatorname{End}\left(M_{R}\right)$ be a monomorphism, with $s M \leq^{e} M$. By GC 2 it follows that $s M=M$. Then by [3, Lemma 3.14], $M_{R}$ is a continuous module. The rest of the proof of (2) follows from Lemma 3.2.

$(2) \Rightarrow(1)$. This implication is obvious. 
COROLLARY 4.3. Let ${ }_{S} M_{R}$ be a bimodule with $S=\operatorname{End}\left(M_{R}\right)$. Then the following are equivalent:

(1) ${ }_{S} M_{R}$ is a $W^{*}$-module, $l_{S}(A)+l_{S}(B)$ is an annihilator for any two relative complements $A$ and $B$ of $M_{R}$, and $M_{R}$ has $G C_{2}$;

(2) $M_{R}$ is a continuous module.

In particular, if $M_{R}$ is of finite uniform dimension, then $S$ is semiperfect.

PROOF. It is clear that every monomorphism $f \in \operatorname{End}\left(M_{R}\right)$ is an isomorphism (due to $\mathrm{GC}_{2}$ and $M$ of finite uniform dimension). Hence, $M$ satisfies the assumptions in Camps and Dicks [2, Theorem 5], and so $\operatorname{End}\left(M_{R}\right)$ is semilocal. Therefore by using [3, Proposition 3.5 and Lemma 3.7], idempotents of $S / J(S)$ lift to idempotents of $S$, and thus $S$ is semiperfect.

LEMMA 4.4. Let ${ }_{S} M_{R}$ be a bimodule such that every finitely generated left ideal of $S$ is a left annihilator of a subset of $M_{R}$, and every closed submodule of $M_{R}$ is a right annihilator of a finite subset of $S$. Then $M$ has condition (1.1).

Proof. Let $A_{1}$ and $A_{2}$ be complements of each other in $M_{R}$. Then by assumption, we have $A_{i}=r_{M}\left(Y_{i}\right)$ for some finite subsets $Y_{i}$ of S. Again by assumption, $S Y_{i}=l_{S}\left(K_{i}\right)$ for some subsets $K_{i}$ in $M_{R}$, where $i=1$,2. Now $S=$ $l_{S}\left(A_{1} \cap A_{2}\right)=l_{S}\left(r_{M}\left(Y_{1}\right) \cap r_{M}\left(Y_{2}\right)\right)=l_{S} r_{M}\left(S Y_{1}+S Y_{2}\right)=S Y_{1}+S Y_{2}$ (due to the assumption and since $S Y_{1}+S Y_{2}$ is finitely generated). Hence $S=l_{S}\left(K_{1}\right)+l_{S}\left(K_{2}\right)=$ $l_{S} r_{M} l_{S}\left(K_{1}\right)+l_{S} r_{M} l_{S}\left(K_{2}\right)=l_{S} r_{M}\left(Y_{1}\right)+l_{S} r_{M}\left(Y_{2}\right)=l_{S}\left(A_{1}\right)+l_{S}\left(A_{2}\right)$. Therefore $M$ satisfies condition (1.1).

LEMMA 4.5. Let ${ }_{S} M_{R}$ be a bimodule and let every idempotent in $\operatorname{End}\left(M_{R}\right)$ be a left multiplication by an element of $S$. If $M_{R}$ is a CS-module, then every closed submodule of $M_{R}$ is a right annihilator of a finite subset of $S$.

Proof. Let $A$ be a closed submodule of $M_{R}$. Then by CS, there exists $f^{2}=$ $f \in \operatorname{End}\left(M_{R}\right)$ such that $A=r_{M}(1-f)=\{m \in M:(1-s) m=0\}=r_{M}(1-s)$, where $(1-s) \in S$.

The following corollary is an immediate consequence of Lemmas 4.4 and 4.5.

COROLLARY 4.6. Let ${ }_{S} M_{R}$ be a bimodule, where $S=\operatorname{End}\left(M_{R}\right)$. Let every finitely generated left ideal of $S$ be a left annihilator of a subset of $M$. Then the following are equivalent:

(1) every closed submodule of $M$ is a right annihilator of a finite subset of $S$;

(2) $M$ is a CS-module.

THEOREM 4.7. Let ${ }_{S} M_{R}$ be a bimodule, where $S=\operatorname{End}\left(M_{R}\right)$. Let every finitely generated left ideal of $S$ be a left annihilator of a subset of $M$. Then the following are equivalent:

(1) $M$ is a CS-module;

(2) $M$ is continuous. 
Proof. By Lemmas 4.4 and 4.5, we have that $M$ has condition (1.1) . By Remark 3.3, $M$ is quasicontinuous. To show that $M$ is continuous, by [3, Lemma 3.14], it is enough to show that every essential monomorphism $s \in S$ is an isomorphism. Let $s \in S$ be a monomorphism, with $s M \leq^{e} M$. By assumption, $S_{S}=l_{S}(X)$ for some subset $X$ of $M$. It follows that $X=0$ and hence $S_{S}=s$. Then $s$ is a split monomorphism, and therefore $s M=M$.

\section{REFERENCES}

[1] V. Camillo, W. K. Nicholson, and M. F. Yousif, Ikeda-Nakayama rings, J. Algebra 226 (2000), 1001-1010.

[2] R. Camps and W. Dicks, On semi-local rings, Israel J. Math. 81 (1993), 203-211.

[3] S. H. Mohamed and B. J. Muller, Continuous and Discrete Modules, Cambridge University Press, Cambridge, 1990.

[4] R. Wisbauer, M. F. Yousif, and Y. Zhou, Ikeda-Nakayama modules, Beiträge Algebra Geom. 43 (2002), no. 1, 111-119.

Mahmoud A. Kamal: Department of Mathematics, Faculty of Education, Ain Shams University, Roxy, Cairo, Egypt

E-mail address: mahmoudkama1333@hotmai 1.com

Amany M. Menshawy: Department of Mathematics, Faculty of Education, Ain Shams University, Roxy, Cairo, Egypt 


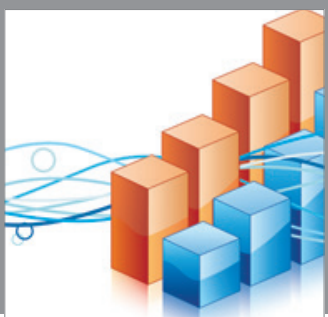

Advances in

Operations Research

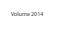

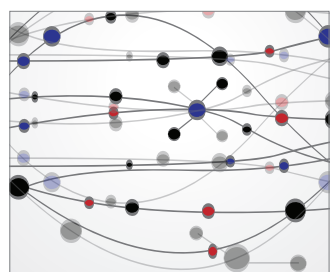

\section{The Scientific} World Journal
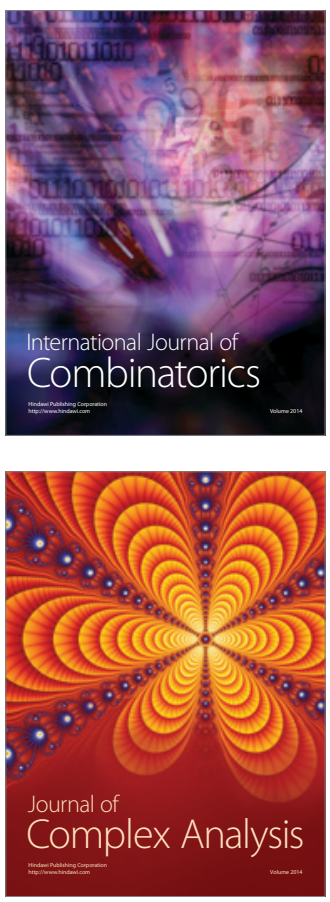

International Journal of

Mathematics and

Mathematical

Sciences
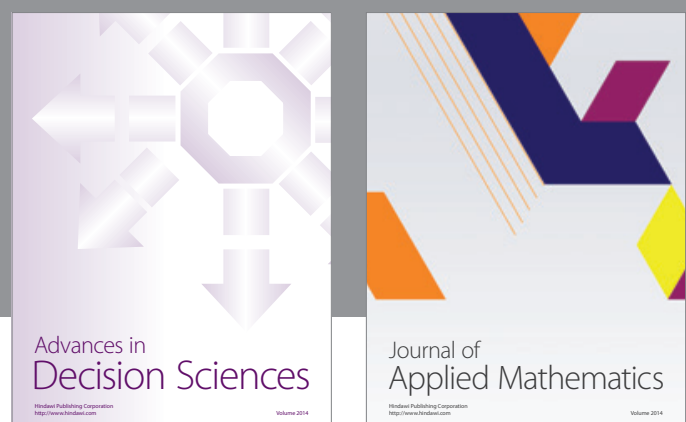

Journal of

Applied Mathematics
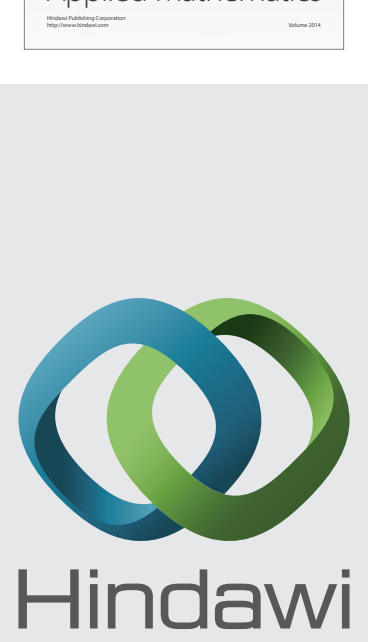

Submit your manuscripts at http://www.hindawi.com
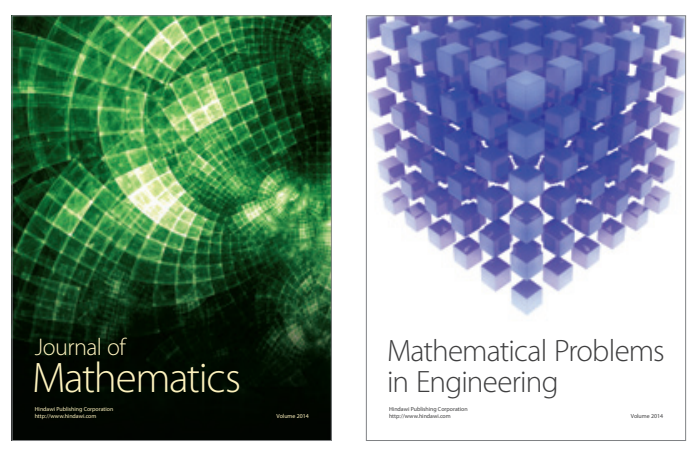

Mathematical Problems in Engineering
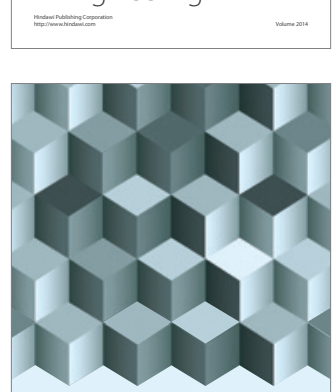

Journal of

Function Spaces
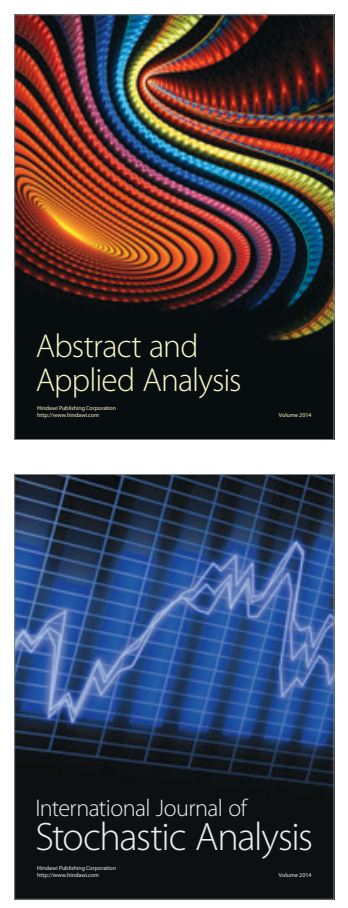

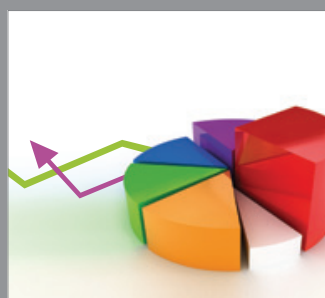

ournal of

Probability and Statistics

Promensencen
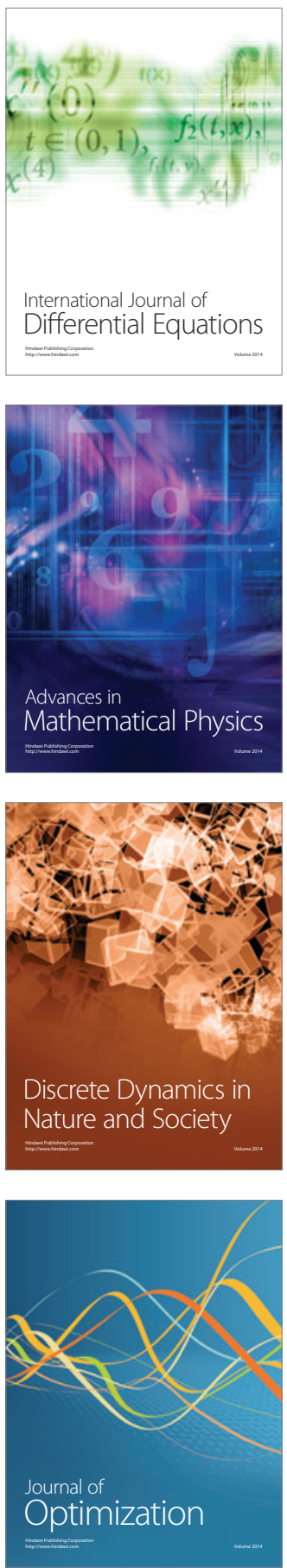